Methods In 6 patients with AOSD according to the Yamagushi criteria of 1992, a therapy with infliximab was started. Malignant and infectious diseases or other inflammatory rheumatic diseases had been excluded. All patients had severe disease with high clinical and serological activity and had initially been treated with high-dose steroids. Patient 1 had a history of 3 years of AOSD with fever, chills, pleural and pericardial effusions and hepatosplenomegaly and had previously been treated with methotrexat and azathioprine. Despite of this, he developed increasing serological signs of inflammation and arthritis of both hips and peripheral joints. The second patient had a history of 5 years of AOSD with oligoarthritis, myalgias and recurrent fever despite of treatment with azathioprine, methotrexat, first administered alone, then in combination with cyclosporine $\mathrm{A}$ and cyclophosphamide pulse therapy. The other four patients had an earlier stage of their disease and did not have prior DMARD treatment apart from the initial steroid therapy. All of our patients with AOSD had polyarthralgias, splenomegaly, continuing fever, leukocytosis, elevated CRP, ESR and ferritin levels. Five patients showed the typical rash, and 3 had polyarticular synovitis. The attempt to reduce immunosuppressive treatment caused a relapse of the disease in all of our patients. All patients received $5 \mathrm{mg} / \mathrm{kg}$ infliximab at week 0,2 , and 6 . Thereafter infliximab infusions were continued at intervals of 6 to 8 weeks depending on the patient's individual disease activity.

Results In all patients, fever, arthralgias, myalgias, splenomegaly and the rash resolved within the first three courses of treatment with infliximab. All serological parameters (CRP, ESR, hyperferritinaemia) returned to normal values. After three courses of infliximab infusions, splenomegaly could not be detected in any of our patients. One patient had still severe pain in the left hip, caused by hip postarthritic osteoarthrosis, requiring hip replacement. Up to now, our patients have been treated between 2 and 18 months. Throughout this treatment period all patients continued to benefit from this therapy with regard to their clinical symptoms, their joint counts and their serological disease activity. One of our patients experienced a moderate infusion reaction during the second treatment. The infusion was discontinued for one hour and could be resumed after that without any problems.

Conclusion In conclusion, all 6 patients with AOSD showed marked improvement of the disease after treatment with infliximab, also in an early stage of AOSD. Our preliminary data suggest the potential therapeutic benefit of anti-TNF treatment in AOSD.

\section{THU0230 ANTI-TNFA THERAPY WITH INFLIXIMAB IN THE TREATMENT OF HLA B27 ASSOCIATED ACUTE ANTERIOR UVEITIS}

${ }^{1} Y$ El-Shabrawi, ${ }^{2} \mathrm{~J}$ Hermann. ${ }^{1}$ Department of Ophthalmology; ${ }^{2}$ Department of Internal Medicine, Karl-Franzens-University Graz, Graz, Austria

\subsection{6/annrheumdis-2001.762}

Background Application of topical corticosteroids is the first-line treatment of anterior uveitis. Its most common form is HLA B27 associated. Of these patients approximately 55\% suffer from additional systemic diseases, such as ankylosing spondylitis or chronic inflammatory bowel disease. In a recent report Brandt, et al. ${ }^{1}$ reported a dramatic response to Infliximab in patients with ankylosing spondylitis. In about 50\% of uveitis patients additional periocular or oral corticosteroids or both are needed because no adequate response to intensive (hourly) topical corticosteroid therapy can be achieved. This together with the fact that recurrent treatment of these young patients with corticosteroids dramatically increases the risk of steroid-induced side effects such as glaucoma, cataract or, in the case of oral or intravenous application, hypertension, diabetes mellitus or osteoporosis new approaches to the therapy of acute uveitis are highly needed.

Objectives To evaluate the potentials of Infliximab, a neutralising Anti-TNFa antibody in the therapy of patients with an acute HLA B27 associated anterior uveitis.

Methods Seven consecutive patients with an acute onset of an HLA B 27 associated anterior uveitis, with at least $3+$ anterior chamber cells were included in this prospective non-comparative case series. Infliximab (Centocor, Malvern, Pa.) at a dose of 10 $\mathrm{mg} / \mathrm{kg}$ body weight given intravenously was used as the sole anti-inflammatory drug.

Anterior chamber cells and flare were evaluated prior to Infliximab treatment and at defined time points after treatment. CRP levels were assessed in all patients prior to intravenous delivery of Infliximab and re-evaluated after one week.

Results Patients were followed for a mean period of $194 \pm 32$ days. No patient treated showed clinical signs of a spondylarthropathy. Seven patients received a single Infiximab infusion of 10 $\mathrm{mg} / \mathrm{kg}$ body weight. A second infusion was administered in one patient 21 days after first dosage due to a reactivation of the uveitis. The median duration $( \pm \mathrm{SD}$ ) of uveitis of $8 \pm 12$ days. All patients responded to Infliximab with an rapid improvement of clinical symptoms a decrease of anterior chamber cells. Only in one patient a total resolution of the uveitis was not achieved using Infliximab.

Conclusion In our study Infliximab proofed to be a powerful therapeutic agent in acute HLA B27 associated uveitis. It therefore seems to be an excellent alternative or an additive to steroid treatment. Only in patients with a systemic sign of an inflammatory disease additional local, systemic low dose corticosteroids or immunosuppressive agents might be needed.

\section{REFERENCE}

1 Brandt J, Haibel H, Cornely D, Golder W, Gonzalez J, Reddig J, Thriene W, Sieper J, Braun J. Successful treatment of active ankylosing spondylitis with the antitumor necrosis factor alpha monoclonal antibody infliximab. Arthritis Rheum. 2000;43(6):1346-52

\section{THU0231 RHEUMATIC DISEASE AS THE CAUSE OF FEVER OF UNKNOWN ORIGIN: AN UPDATE OF CLASSICAL DATA}

${ }^{1} \mathrm{MA}$ Oztürk, ${ }^{1} \mathrm{~S}$ Kiraz, ${ }^{1} \mathrm{I}$ Ertenli, ${ }^{2} \mathrm{O}$ Uzun, ${ }^{1} \mathrm{~S}$ Apras, ${ }^{1} \mathrm{M}$ Çalgüneri, ${ }^{2} \mathrm{~S}$ Unal. ${ }^{1}$ Rheumatology; ${ }^{2}$ Infectious Disease, Hacettepe University School of Medicine, Ankara, Turkey

\subsection{6/annrheumdis-2001.763}

Background Fever of unknown origin (FUO) remains one of the major diagnostic challenges for the clinician. Although infection, malignancy and collagen vascular disease are accepted the 3 most important causes of FUO, the relative importance of these major categories might have changed because of improvements in serodiagnosis, culture techniques and radiologic imaging modalities. Rheumatic diseases not uncommonly present with fever.

Objectives Our objective was to make an uptake of our knowledge regarding causes of FUO in our hospital together with more detailed analysis of collagen vascular and granulomatous disorders presenting with FUO. 